\title{
Comparación de tecnologías para la localización de conexiones ilegales de agua potable
}

Comparison of technologies for the location of illegal drinking water connections

Recibido: noviembre 10 de 2019 | Revisado: diciembre 13 de 2019 | Aceptado: enero 10 de 2020

\author{
STEFAn ZiemendorfF ${ }^{\text {IA }}$ \\ Michael Kerstingr,
}

\begin{abstract}
Resumen
Uno de los principales problemas que enfrentan las entidades prestadoras es el hurto de agua potable, comúnmente denominado clandestinaje. Una de las formas más comunes del hurto de agua se produce a través de instalaciones ilegales, las cuales hasta hace poco han sido de difícil detección y localización. El presente artículo compara las diferentes tecnologías actualmente disponibles para la localización de instalaciones ilegales, destinadas a hurtar agua potable. Para ello expone las ventajas, desventajas, alcances y límites de cada una de las tecnologías identificadas para que las entidades prestadoras puedan escoger las más adecuadas para sus condiciones locales.
\end{abstract}

Palabras clave: agua potable, hurto de agua, conexiones ilegales, localización de conexiones ilegales

\begin{abstract}
One of the main problems facing utilities is the unauthorized consumption of drinking water, commonly called clandestine appropriation or water theft. One of the most common forms of water theft occurs through illegal installations, which until recently have been difficult to detect and locate. This article compares the different technologies currently available for locating illegal installations, designed for clandestine appropriation of drinking water. To this end, it exposes the advantages, disadvantages, scope and limits of each of the identified technologies, so that the utilities can choose the most appropriate for their local conditions.
\end{abstract}

Key words: drinking water, water theft, illegal connections, location of illegalconnections

\footnotetext{
(C) Los autores. Este artículo es publicado por la Revista Campus de la Facultad de Ingeniería y Arquitectura de la Universidad de San Martín de Porres. Este artículo se distribuye en los términos de la Licencia Creative Commons Atribución No-comercial - Compartir-Igual 4.0 Internacional (https://creativecommons.org/licenses/ CC-BY), que permite el uso no comercial, distribución y reproducción en cualquier medio siempre que la obra original sea debidamente citada. Para uso comercial contactar a: revistacampus@usmp.pe.
} 


\section{Introducción}

El hurto de agua potable es, junto con las fugas de agua y el subregistro de medidores, una de las causas más importantes de elevados niveles de agua no facturada (ANF) en muchas entidades prestadoras de servicios de saneamiento, con efectos negativos tanto en el ámbito comercial como operativo. Las más frecuentes formas de hurto son mediante instalaciones ilegales permanentes, instaladas normalmente de tal forma que permanecen ocultas durante mucho tiempo y pueden ser encontradas solamente por casualidad o con esfuerzos proactivos de las entidades prestadoras.

El presente artículo trata de estos esfuerzos proactivos que consisten en métodos de detección y localización de estas instalaciones ilegales, enfocados en instalaciones de PVC y HDPE, ya que estos materiales son las de mayor uso en los países de América Latina y el mundo. Estos métodos son en su mayoría insuficientemente conocidos, especialmente en lo que a la factibilidad de su aplicación en el campo bajo condiciones diferentes se refiere, conllevando en muchos casos a la adquisición de equipos costosos de aplicación muy limitada o incompleta. Por ello se presenta aquí un estudio comparativo de aquellos métodos, que bajo circunstancias bien definidas, pueden ayudar a las entidades prestadoras a disminuir sustancialmente las instalaciones ilegales de manera costo-eficiente.

\section{Método}

El hurto de agua potable se realiza de muchas formas diferentes, como a través de la manipulación de medidores de consumo (Figura 1 - A y B), la reapertura (o reconexión) ilegal de conexiones previamente cerradas (cortadas), por ejemplo, por la falta de pago del servicio (Figura $1-C$ ) y a través de instalaciones ilegales, con frecuencia también llamadas clandestinas o fraudulentas (Figura 1 D, E y F).

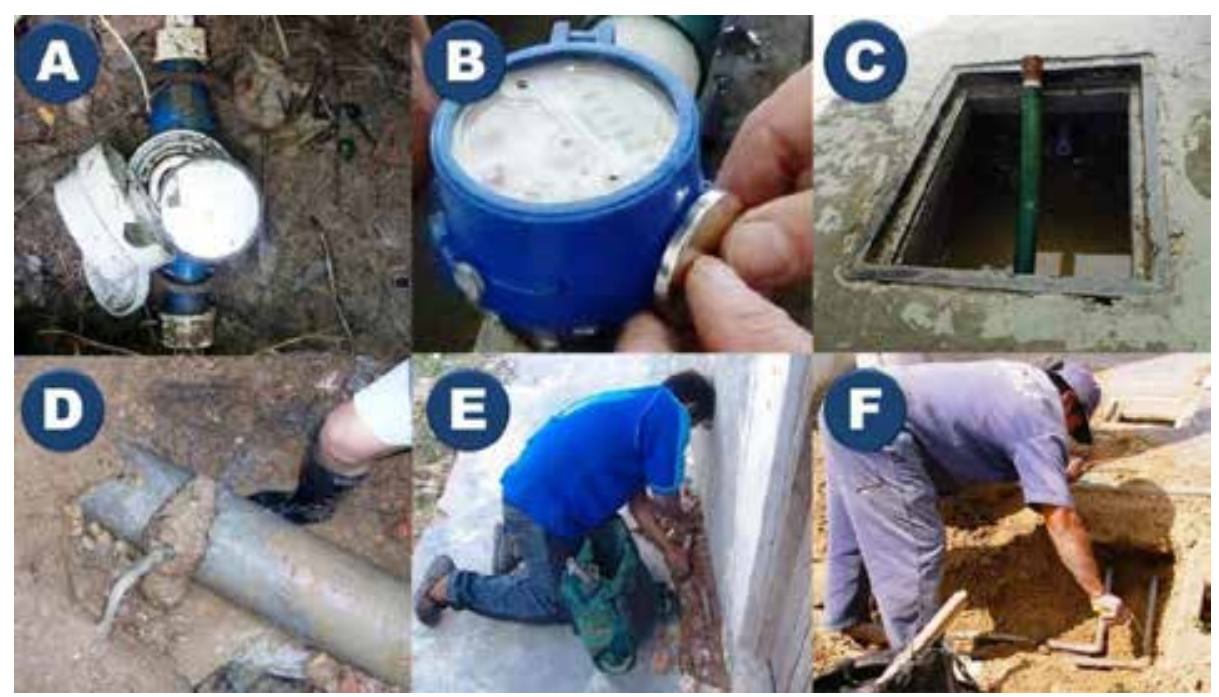

Figura 1. Algunas formas de hurto de agua potable: A - Medidor manipulado, separando su relojería del cuerpo; B - Medidor paralizado con un imán; C - Reconexión ilegal de una conexión previamente cerrada; D - Toma clandestina a una red pública; E - Conexión ilegal a una conexión legal vecinal por la pared del inmueble (derivación); F - Conexión ilegal a la propia conexión legal (bypass). 
Las formas de hurto de agua han sido clasificadas por el tipo de usuario que lo realiza y la forma de hurto (Alva et al., 2019, cap. 3) y son resumidas en la Tabla 1.

Tabla 1

Formas de hurto por tipo de usuario. Aquellas formas de hurto que requieren ser localizadas con los métodos analizados en el presente artículo son marcadas en verde, las formas de hurto según tipo de usuario que no existen están marcadas en rojo, y en amarillo aquellas que necesitan ser combatidas de otra forma.

\begin{tabular}{|l|l|l|l|}
\cline { 2 - 4 } \multicolumn{1}{c|}{} & \multicolumn{3}{c|}{ Tipo de } \\
\multicolumn{1}{c|}{ Formaario }
\end{tabular}

La presente publicación no abarcará todas estas formas principales de hurto de agua potable posibles, como el hurto a través de la manipulación de medidores (Ziemendorff et al., 2017) o a través de la reaperturailegaldeunserviciopreviamente clausurado o cortado (Ziemendorff et al., 2018) sino aquellos que se realizan a través de instalaciones ilegales en la vía pública (Figura 2), específicamente: (I) Conexión ilegal directa a la red pública; Estas son las frecuentemente llamadas conexiones clandestinas. La conexión puede ser la única en el caso de usuarios que no son clientes. También puede ser una conexión adicional a la legal en el caso de los clientes activos o inactivos.
(II) Derivación ilegal de una conexión vecinal; nuevamente puede tratarse de la única conexión del usuario que no es cliente o de una derivación que es adicional a la conexión legal de los clientes activos o inactivos. (III) Bypass; también se trata de una derivación a una conexión legal como en el caso anterior, pero a diferencia de esta, la derivación no se hace a una conexión vecinal, sino a la conexión del mismo cliente para evitar que el agua pase por el medidor de consumo. Este tipo de instalación solo se halla en clientes activos con medidor y en ocasiones también en clientes inactivos (cortados), pero no en no clientes o en clientes sin medidor. 


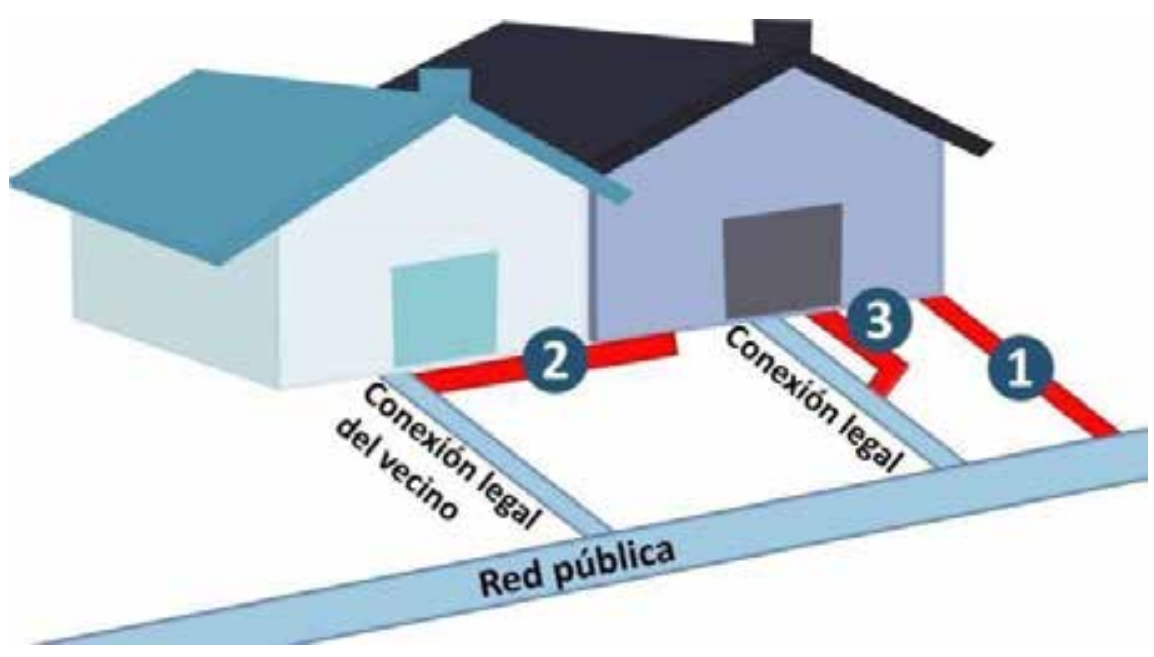

Figura 2. Instalaciones ilegales de agua potable en la vía pública: 1 Conexión ilegal directa a la red pública; 2 - Derivación ilegal de una conexión vecinal; 3 - Bypass

\section{Metodología de comparación}

Los métodos de localización de instalaciones ilegales serán analizados uno por uno bajo los siguientes aspectos: (i) Alcances; se analizaría cuál de las tres variantes de instalaciones ilegales (conexión a la red, conexión a otra conexión, bypass) se puede localizar o no con cada método. (ii) Limitaciones; se analizará cuáles son las condiciones locales bajo las cuales se puede o no localizar instalaciones ilegales con cada método. (iii) Probabilidad de localizaciones erróneas; se analizará, para cada método, en qué casos y con qué frecuencia relativa pueden ocurrir detecciones de instalaciones ilegales que no son tales (falsos positivos).

1. Margen de error: Se analizaría la precisión con la cual se detectan instalaciones ilegales y cuáles son los factores que influyen.

2. Facilidad de uso: Se analizaría con qué necesidad de tiempo, equipos y/o materiales adicionales puede realizarse la localización de instalaciones ilegales.

3. Inversión requerida: Se compararán los métodos con referencia a los costos de adquisición.

4. Recursos humanos requeridos: Se analizará la cantidad de personal requerido y sobre todo el grado de capacitación y experiencia que este requiere.

El análisis tomará en cuenta por un lado publicaciones previas, experiencias propias con los equipos, y experiencias de campo de algunas entidades prestadoras, especialmente de Perú, con los métodos.

Métodos de localización de instalaciones de agua potable incluidos en el presente estudio

Los métodos que se han analizado y comparado entre sí, bajo los aspectos indicados, son los que se detallan a continuación y que son mostrados en la Figura 3. 


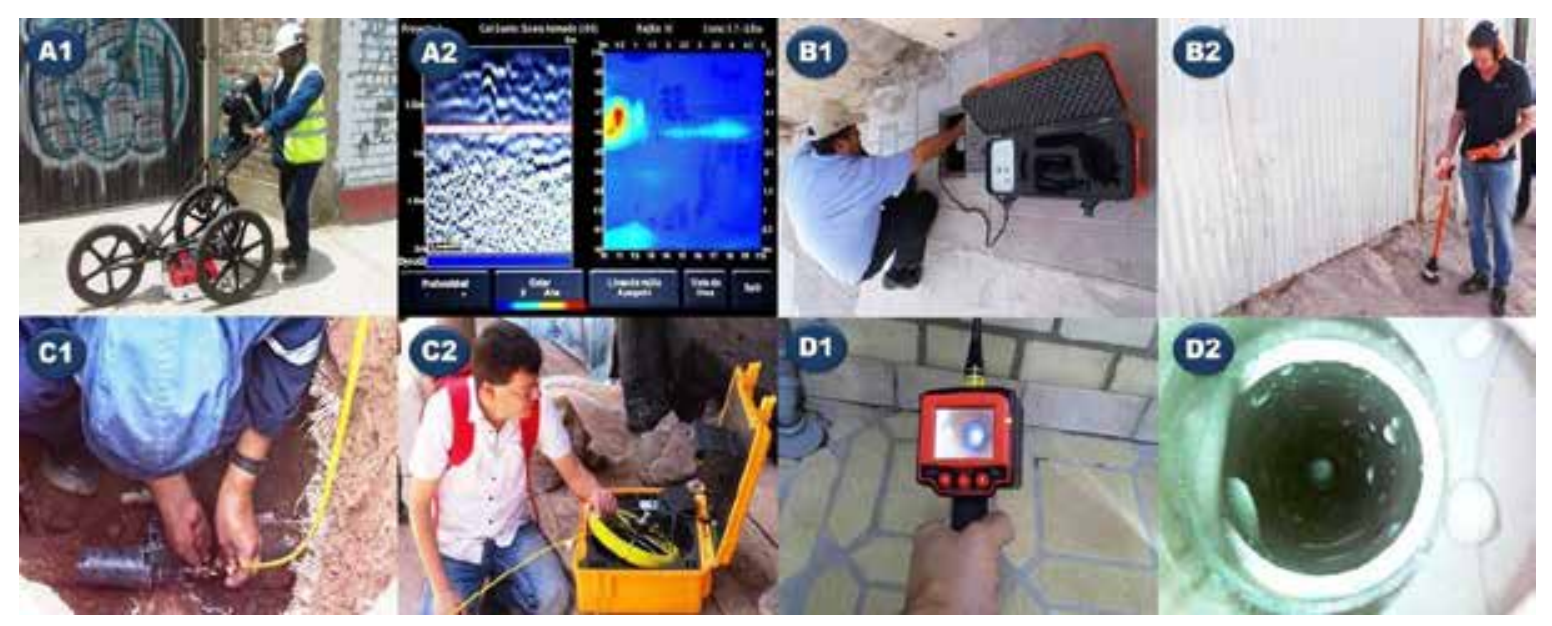

Figura 3. Métodos incluidos en el presente estudio: A1 - Georradar en operación (cortesía: Teony Alva); A2 - Pantalla del georradar mostrando una tubería; B1 - Método electroacústico, conexión de un oscilador; B2 - Método electroacústico, localización del sonido con un geófono; C1 - Cámara endoscópica es introducida a la red pública; C2 - Revisión de la pantalla de la cámara endoscópica para redes; D1 - Cámara endoscópica para conexiones; D2 - Foto endoscópica de una derivación a un conexión domiciliaria.

El georradar capta los reflejos que se producen en las diferentes capas del terreno, las cuales se producen acorde a la conductividad del material. A partir del tiempo de tránsito entre la señal de transmisión y la señal del reflejo, calcula la profundidad donde se produce el reflejo. Para ello el equipo es empujado lentamente sobre la superficie del subsuelo a escanear (Figura 3-A1). Los resultados del escaneo son mostrados en una pantalla incorporada al equipo (Figura 3 - A2). Esta tecnología se usa desde hace algunos años para la detección de conexiones ilegales en varios países como en Chile, Colombia y Perú.

El método electroacústico, consiste en ubicar instalaciones ilegales induciendo un sonido en las tuberías de agua, el cual se propaga a través de las tuberías a cierta distancia y puede ser localizado desde la superficie con un geófono (Figura 3 B2). El sonido es inducido mediante un oscilador que es colocado en algún punto de la tubería, como en un hidrante, pero más frecuentemente en una conexión.
Hay dos tipos de osciladores: el primero se acopla directamente a la columna de agua y, al cerrarse, produce un impulso hidráulico que se propaga en el sistema produciendo sonidos audibles. El segundo tipo de oscilador también se coloca en algún punto de la tubería (Figura 3 - B1) y somete las tuberías a vibraciones al golpearlas desde afuera, como un martillo eléctrico. El método es usado a nivel mundial desde muchos años para la localización de tuberías plásticas.

Inspección interna de redes con cámaras endoscópicas: consiste en la introducción de cámaras de video, previstas de diodos LED con cables en las redes de agua potable desde cualquier punto, como desde una válvula, aunque para la localización de conexiones ilegales en sitios definidos muchas veces es necesario perforar un hueco en la tubería para tener un punto de acceso cercano (Figura 3-C1), el mismo tiene que ser reparado posteriormente. Estos cables son muy rígidos para que permitan conducir la cámara por largos trayectos 
de hasta 60 metros, empujándolas. En las redes de agua se usa tanto para la detección de fugas como para la detección de instalaciones ilegales, para lo cual es necesario cortar el servicio en la zona sondeada previamente, aunque también hay sistemas que funcionan bajo presión.

Especialmente para la localización de conexiones ilegales se prefiere, sin embargo, sistemas que operan con la tubería vacía, debido a la más fácil operación y mejor visibilidad. Las imágenes captadas por la cámara son revisadas en una pantalla conectada al cable y pueden ser grabadas (Figura 3 C2).

Inspección interna de conexiones domiciliaras con cámaras endoscópicas, consiste en la introducción de cámaras de video, previstas de diodos LED, con cables flexibles en las conexiones domiciliarias de agua potable. Ello evita que puedan ser empujadas a muy largas distancias, lo cual tampoco es necesario ya que las conexiones raramente pasan los siete $\mathrm{u}$ ocho metros de largo, pero sobre todo porqué eventuales bypass o derivaciones a la conexión se encuentran más cerca al predio que a la red. A la vez tanto la cámara como el cable son necesariamente muy finos para que pueden penetrar tubos a partir de un diámetro de $1 / 2$ "se introduce por la caja portamedidor hacía la red, pudiendo detectar y localizar derivaciones de la conexión y bypass. Las imágenes captadas por la cámara son revisadas en una pantalla incorporada al equipo (Figura 3 - D1 y D2), aunque también existen algunos que pueden ser conectados a celulares. La razón de tratar este método aparte del anterior consiste en que los resultados de los factores evaluados son sumamente diferentes para cada tipo de cámara endoscópica y que pueden ser usadas tanto en forma combinada como también cada uno por sí mismo en combinación con otros métodos.

\section{Resultados}

El georradar puede localizar tanto tuberías enterradas como, con frecuencia, las zanjas excavadas para enterrarlas, debido a que estas presentan con frecuencia una densidad diferente al terreno natural. Sin embargo, no puede distinguirse con este equipo dos tuberías cuando estas están muy cercanas entre ellas o la tubería que está justo por debajo de la caja del medidor, porque la señal electromagnética no penetra bien a través de este vacío. Sin embargo, Bypasses presentan con frecuencia justo estas características, y son por ello normalmente no detectables con el georradar. En el caso del método electroacústico la situación es parecida, el sonido inducido a la tubería llega a la superficie indistintamente si se trata de un tubo legal o uno ilegal, y cuando van cerca el uno del otro, como ocurre con los bypasses, es imposible localizarlo con ese método, mientras con tubos separados, como es normalmente el caso con conexiones directas a la red o derivaciones de otras conexiones, son localizables con facilidad. Tanto este método como el georradar si pueden hallar bypasses en aquellos casos aislados en las cuales el bypass fue instalado apartándose mucho de la conexión legal. En el caso de las cámaras endoscópicas es evidente que las cámaras previstas de revisar las redes solo pueden encontrar tomas ilegales en estas y equivalentemente las cámaras para conexiones solo hallan las derivaciones producidas en estas. 
En resumen, ninguno de los diferentes métodos puede localizar los tres tipos de instalaciones ilegales. Dentro de las limitaciones de los métodos tenemos que el georradar no funciona de manera satisfactoria en subsuelos cuya conductividad eléctrica es muy alta, en los cuales, en consecuencia, se pierde la señal y no produce los rebotes necesarios para su funcionamiento correcto. Estos suelos son: salinos, sódicos, muy arcillosos y con gran humedad (Doolittle et al., 2007). Otros tipos de subsuelos pueden ser igualmente problemáticos cuando su conductividad es igual o muy parecida a aquella del tubo enterrado, como ocurre en el caso de tubos de PVC enterrados en arena de cuarzo. El georradar tampoco puede localizar aquellas tuberías que se encuentran enterradas debajo de superficies no planas, en las cuales la señal no penetra uniformemente o inaccesibles para el equipo como en bordes de casas, escaleras o veredas. En la práctica esta es una limitación bastante importante ya que las conexiones ilegales son instaladas con frecuencia en estos lugares porque se puede esconder más fácilmente las evidencias que deja la instalación que en plena vereda (huellas de concreto fresco / ralladuras).

El método electroacústico es más difícil incluso imposible de aplicar en subsuelos que conducen muy bien sonidos, esto es en subsuelos muy compactos y rocosos. Bajo estas condiciones puede ocurrir con frecuencia que el sonido transmitido a través de las tuberías ya no sea distinguible del mismo sonido transmitido a través del subsuelo, aumentándose los falsos positivos y el margen de error hasta quedar totalmente inútil. Otra limitación consiste en que, para que los osciladores que inducen el sonido a las tuberías de agua funcionen requieren un acceso a la red. Ello en zonas urbanas es normalmente fácil, ya que se les puede conectar a las conexiones de agua en las cajas portamedidor. Sin embargo hay predios muy grandes, por ejemplo en áreas industriales donde hay cientos de metros de una conexión a la otra. Bajo estas condiciones el uso de los equipos de localización es sumamente limitado a distancias unos 50 a 60 metros contados a partir del punto en el cuál es instalado el oscilador de golpes de ariete y a la mitad para el oscilador tipo martillo eléctrico. Otra limitación adicional solamente válida para el oscilador que usa golpes de ariete, es que este equipo solo funciona en la horas de servicio y cuando la presión está encima de 15 mca (21 PSI aprox.) y llega a mayores distancias cuando la presión está por encima de 25 mca (33 PSI aprox.). Finalmente, independiente del tipo de oscilador que se usa no se recomienda su uso en zonas muy ruidosas en la horas de mayor ruido.

Un limitante de las cámaras endoscópicas para redes es la dificultad de evitar que la cámara no pueda captar las imágenes cuando se ensucia o se moja. Esto en ciertos casos es sumamente difícil de asegurar, cuando se trate de redes con presencia de incrustaciones de cal o presencia de otras partículas de origen orgánico o inorgánico. Esto puede ser solucionado si la cámara endoscópica es introducida primero en un punto, y luego sacada, limpiada e introducida en otro punto a la distancia máxima permitida y luego llevada hacia atrás con menor posibilidad de ensuciarse. Cabe de indicar que esto requiere un mayor esfuerzo por garantizar dos puntos accesibles a la red. No solucionable con el mismo método es que en varios puntos 
de la red, específicamente en zonas sin pendiente de la red, el agua tiende a estancarse. Ello se debe a que las tuberías de agua se hunden en forma desigual, aunque por pocos centímetros, por el paso de los años y por la presión de las capas superiores a ellos. En estos puntos el agua puede estancarse con el resultado de que algunas gotas se quedan en la cámara.

El principal y bastante frecuente limitante para el uso de cámaras endoscópicas para la inspección de conexiones son los obstáculos en las conexiones de agua, que no permiten su introducción. Su presencia depende de la forma que las conexiones fueron ejecutadas. Cuando sean de material rígido (PVC) tienen con frecuencia dos codos de $90^{\circ}$ seguidos para bajar la tubería del nivel de la vereda hacía la red matriz. Si bien el cable conductor es lo suficiente flexible para pasar por curvas de hasta $90^{\circ}$ si son del mismo tubo, ello no es el caso cuando se trata de accesorios con bordes. En los últimos años se ha hecho más frecuente la ejecución de conexiones con tubería flexible de polietileno de alta densidad, que facilitan la inspección interna de las conexiones con cámaras endoscópicas, sin embargo la gran mayoría de las conexiones instaladas aún son hechas con material rígido.

\section{Probabilidad de localizaciones erróneas}

De los métodos analizados el georradar presenta las mayores probabilidades de conllevar al operador a una interpretación errónea de los datos, en el sentido de que se identifica una instalación como ilegal que no lo es. Ello se debe a que con el georradar no se pueden distinguir tuberías de agua de otros objetos enterrados, por ejemplo, de ductos de otros servicios como gas, luz y telecomunicaciones. También la presencia de desagües pluviales, encofrados de hierro de construcción, raíces o muchas piedras grandes, aumenta la complejidad de la interpretación de los datos de tal forma que aún con mucha experiencia se pueden dar muchos falsos positivos hasta el extremo que en subsuelos muy comprometidos ello se vuelve una limitación.

Con el método electroacústico no pueden darse falsos positivos por la presencia de otros servicios, salvo que estos estén en contacto con la tubería de agua de tal forma que el sonido pueda pasar a ellos y cuando estos ductos propagan mejor el sonido que la tubería de agua por ser de metal (ductos de gas, por ejemplo). Otras posibilidades de localizaciones erróneas pueden darse por la misma razón, objetos que son muy buenos conductores acústicos (en concreto, piedra o metal) llevan el sonidoa otro lado, lo cual es sin embargo poco frecuente.

En el caso de las cámaras endoscópicas para redes, estas dejan muy poco margen de interpretación $y$ pueden inducir a localizaciones erróneas solamente en muy contados casos, por ejemplo, cuando una conexión ilegal ya fue hallada y eliminada anteriormente, pero se dejó la abrazadera y es nuevamente detectada. Otro caso es en tramos con muchas conexiones de agua seguidas, pero de los cuales no todos están conectados directamente a la red, sino dos o más conexiones usan una sola toma. Si el catastro de la entidad prestadora no ha registrado correctamente esta información es prácticamente imposible distinguir entre tomas legales e ilegales en estos tramos. 
Con las cámaras endoscópicas para conexiones es posible hallar una derivación legal a una conexión legal y en caso que el catastro no haya registrado este hecho correctamente, pueda ser tomada erróneamente como una derivación ilegal, pero se trata nuevamente de casos muy aislados.

\section{Margen de error de la localización}

El georradar, en caso de que la identificación de una tubería ilegal ha sido correcta, tiene un margen de error mínimo con una precisión de pocos centímetros. En el caso del método electroacústico el margen de error de la localización es un poco mayor y depende principalmente de la profundidad de la tubería ilegal localizada - si está se encuentra cerca de la superficie (menos de medio metro) el margen de error no es mayor a 20 centímetros, pudiéndose aumentar hasta un margen de error de medio metro para tuberías enterradas a la profundidad de la red pública (entre 1 metro a 1.2 metros).

En el caso de ambos métodos endoscópicos el margen de error es mínimo, debido a que los cables conductores cuentan con reglas impresas en ellas, el cual es luego medido en la superficie con ruedas de medición. Por la mayor longitud de los tramos recorridos en el caso de las cámaras endoscópicas para redes, el margen de error puede llegar a 10 o 20 centímetros, mientras en el caso de las cámaras para conexiones domiciliarias supera pocas veces los cinco centímetros. Esto si puede cambiar en aquellos casos donde los operarios desconocen la ubicación y dirección precisa de las tuberías inspeccionadas, cuando el margen de error puede aumentar considerablemente y superar un metro.

En resumen, los márgenes de error son negables en los casos del georradar y las cámaras endoscópicas (en ese caso solo cuando se conoce la ubicación precisa de la tubería legal), mientras en el caso del método electroacústico es a veces necesario ampliar la excavación para descubrir la instalación ilegal.

\section{Facilidad de uso de los métodos}

La rapidez de la localización de una conexión ilegal existente con el georradar depende en alto grado de las condiciones ya indicadas en el apartado de limitaciones. Si la calidad del subsuelo es poco favorable, pero aún es posible la localización se tiende a demorar mucho más porque hay que calibrar el equipo muy exactamente. Esto se hace en una conexión cuya ubicación es conocida, pasando el georradar con diferentes configuraciones por encima de ella, hasta que se obtenga un resultado óptimo. Otro factor de demora puede ocurrir cuando se cuenta con varias detecciones de anomalías y se requiere confirmar cuál de las anomalías empalma con la red de agua (y que por ello es una conexión de agua). Para ello es necesario conocer la ubicación de la red y realizar el escaneo con "método de rejilla", para lo cual se empuja la careta de forma paralela y transversal varias veces entre la red matriz y el predio. La procesadora interna combina las múltiples franjas escaneadas a una imagen 3D, que puede mostrar hacía donde va cada anomalía, según su respetiva profundidad. Finalmente, el equipo tiene que ser armado y desarmado en cada lugar y con todo ello es muy difícil superar el escaneo de cinco predios por día, exitosos o no. 
La facilidad de uso del método electroacústico depende de las condiciones ya mencionadas en la parte de las limitaciones, cuanto menor es la propagación de sonidos en el subsuelo más rápida es la localización. La demora de la localización propiamente dicha depende adicionalmente mucho de la longitud de la frente (o de los frentes) del predio a inspeccionar. La instalación del oscilador tipo martillo eléctrico no demora más que dos minutos, la del oscilador con golpes de ariete aproximadamente el doble. Con todo ello es posible localizar (o descartar la existencia) de hasta 10 instalaciones ilegales por día, siempre y cuando se realiza un trabajo bien programado.

De los métodos analizados aquel que mayores dificultades en su aplicación presenta es el método con cámaras endoscópicos para redes, lo cual se debe a que se requieren puntos de acceso directo a la red pública de agua para introducir las cámaras. Ello implica que en la mayoría de los casos hay que romper la capa (asfáltica u otra) de las pistas, excavar hasta llegar a la red y perforar a la red para tener un acceso. Posterior a la inspección es preciso reparar la tubería de agua, de tal forma que no se ocasione una fuga, volver a llenar el hueco dejado por la excavación y reponer la pista. Costos, esfuerzos y el tiempo necesario para ello superan muchas veces los beneficios. Solo en ciertos casos, cuando hay presencia de cámaras de válvulas que permiten un acceso directo a la red, sin los trabajos mencionados, esto no es una limitación. Sin embargo, en casi todas las entidades prestadoras, las válvulas de las redes no cuentan con cajas accesibles. Si los trabajos preparatorios y posteriores son hechos de forma independiente de la inspección propiamente dicho es posible inspeccionar unos cinco tramos por día.

Las cámaras endoscópicas para conexiones son de fácil transporte y uso. La principal dificultad y demora en el uso es que es necesario cerrar el circuito de agua en el sector donde se usa, lo cual obviamente no es necesario en momentos de desabastecimiento. Desarmar el medidor y sus accesorios en la caja portamedidor e introducir la cámara dificultosamente demora más de un minuto. Con todo ello es posible revisar hasta 20 conexiones diarias.Como se nota se trata, por lo menos para los tres primeros métodos y especialmente para el equipo georradar, de inversiones de importancia que hacen necesario una evaluación previa de las condiciones locales para poder conocer si los equipos podrán ser usados para el fin que fueron adquiridos.

Adicionalmente, elgeorradaresun equipo complejo en su operación y calibración, pero sobre todo en la correcta interpretación de los datos obtenidos. Para ello, se requiere un personal altamente capacitado en el uso de la correcta configuración del equipo, mientras para la correcta interpretación de los datos obtenidos bajo las condiciones locales específicas se requiere personal experimentado en el uso del georradar. El georradar puede ser en teoría operado por una sola persona; sin embargo, en la práctica siempre se cuenta como mínimo con un auxiliar que ayuda en el armado y traslado del equipo, en la eliminación de obstáculos y en ocasiones para garantizar la seguridad, ya que se trata de un equipo bastante costoso.

El método electroacústico requiere siempre de dos operarios, aquel que opera 
el oscilador, para lo cual no se requiere mayor capacitación ni experiencia y aquel que opera el geófono. Especialmente los geófonos de última generación si requieren algo de capacitación previa para poder aprovechar sus funcionalidades completas, pero sobre todo se requiere de mucha experiencia práctica en el uso y de entrenamiento del oído, lo cual se podría hallar con frecuencia en operarios ya experimentados con equipos para la localización de fugas.

Las cámaras endoscópicas de inspección de redes requieren de por si un mínimo de dos operarios, aquel que introduce y avanza la cámara hacía la red y aquel que revisa la pantalla. Normalmente se cuenta con, por lo menos, un auxiliar adicional a cargo de la excavación y el tapado del acceso a la red, así como de la perforación y reparación posterior del acceso al tubo. La operación propiamente dicha del equipo es sumamente sencilla y no requiere mayor capacitación ni experiencia, aunque si algo más que las cámaras para conexiones.

Para la operación de las cámaras endoscópicas de inspección de conexiones no se requiere más que una persona. No se requiere mayor capacitación ni experiencia para operar el equipo.

\section{Discusión}

Los resultados se resumen en la Tabla 2. Muestran que no existe un método único para la localización de instalaciones ilegales, sino que el método preferible depende de muchos factores.

\section{Tabla 2}

Resumen de factores evaluados por método. Los campos rojos señalan factores desfavorables para el método, los verdes factores favorables y los amarillos factores intermedios

\begin{tabular}{|c|c|c|c|c|c|}
\hline & & Georradar & $\begin{array}{l}\text { Método electro- } \\
\text { acústico }\end{array}$ & $\begin{array}{c}\text { Cámaras } \\
\text { endoscópicas para } \\
\text { redes } \\
\end{array}$ & $\begin{array}{c}\text { Cámaras } \\
\text { endoscópicas para } \\
\text { conexiones } \\
\end{array}$ \\
\hline \multirow{3}{*}{$\frac{\mathscr{Z}}{\tilde{Z}}$} & $\begin{array}{c}\text { Conexión ilegal a la } \\
\text { red }\end{array}$ & Factible & Factible & Factible & No factible \\
\hline & Derivación & Factible & Factible & No factible & Factible \\
\hline & Bypass & Limitado & Limitado & No factible & Factible \\
\hline \multirow{3}{*}{\multicolumn{2}{|c|}{ Limitaciones }} & $\begin{array}{l}\text { Subsuelos con mucha } \\
\text { conductividad (salinos, } \\
\text { sódicos, arcillosos y } \\
\text { húmedos) }\end{array}$ & $\begin{array}{l}\text { Oscilador de golpes } \\
\text { de ariete solo } \\
\text { mientras hay } \\
\text { servicio y si la } \\
\text { presión es mayor a } \\
15 \text { ma }\end{array}$ & \multirow{3}{*}{$\begin{array}{l}\text { Peligro que la cámara } \\
\text { se ensucie o moje }\end{array}$} & \multirow{3}{*}{$\begin{array}{l}\text { Obstáculos en las } \\
\text { conexiones que no } \\
\text { permiten } \\
\text { introducción de la } \\
\text { cámara (codos de } \\
\left.90^{\circ}\right)\end{array}$} \\
\hline & & & Zonas muy ruidosas & & \\
\hline & & $\begin{array}{lc}\text { Subsuelos } & \text { con } \\
\text { conductividad } & \text { muy } \\
\text { parecida al tubo } & \\
\end{array}$ & $\begin{array}{l}\text { Puntos de acceso a la } \\
\text { red muy distantes }\end{array}$ & & \\
\hline \multicolumn{2}{|r|}{ Margen de error aprox. } & $5 \mathrm{~cm}$ & 20 a $50 \mathrm{~cm}$ & 10 a $20 \mathrm{~cm}$ & $5 \mathrm{~cm}$ \\
\hline \multicolumn{2}{|r|}{$\begin{array}{c}\text { Probabilidad de falsos } \\
\text { positivos }\end{array}$} & $\begin{array}{l}\text { Dependiendo de las } \\
\text { condiciones mediana a } \\
\text { alta }\end{array}$ & $\begin{array}{l}\text { Dependiendo de las } \\
\text { condiciones baja a } \\
\text { media }\end{array}$ & Muy baja & Muy baja \\
\hline \multirow{2}{*}{ 茎 } & $\begin{array}{c}\text { Facilidad comparativa } \\
\text { de uso }\end{array}$ & Mediano & Fácil & Muy difícil & Muy fácil \\
\hline & Rendimiento & Hasta 5 predios/día & Hasta 10 predios/día & Hasta 5 tramos/día & $\begin{array}{c}\text { Hasta } 20 \\
\text { conexiones/día }\end{array}$ \\
\hline \multicolumn{2}{|r|}{ Inversión requerida } & US\$ 20,000 a 50,000 & US $\$ 7,000$ a 10,000 & US\$ 4,000 a 8,000 & US\$ 300 a 800 \\
\hline \multirow{2}{*}{ 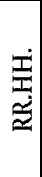 } & $\begin{array}{c}\mathrm{N}^{\circ} \text { de operarios } \\
\text { necesarios }\end{array}$ & $1-2$ & 2 & $2-3$ & 1 \\
\hline & $\begin{array}{c}\text { Capacitación previa } \\
\text { necesaria }\end{array}$ & Mucha & $\begin{array}{c}\text { Operario 1: poca } \\
\text { Operario 2: mediana }\end{array}$ & Poca & Poca \\
\hline
\end{tabular}


Aunque también depende en alto grado de las condiciones locales y circunstanciales, queda claro que el esfuerzo relativo que se requiere para revisar si un predio cuenta con una instalación ilegal o no, es tan demandante en tiempo, recursos humanos y otros, que una pre identificación de predios sospechosos de hurto (Alva et al., 2019, cap. 4) y la exclusión de otras causas que puedan haber llevado a una sospecha no justificada (Ibíd., cap. 5) se hace siempre recomendable. Una excepción a esta regla puede presentar una revisión sistemática de todas las conexiones con cámaras endoscópicas en aquellos sitios donde son aplicables y que presentan altos índices de hurtos. Otro punto clave que indican los resultados del análisis es que, para una estrategia integral de localización masiva de instalaciones ilegales, casi siempre se requiere la aplicación combinada de dos o más métodos.

Finalmente, lo que se desprende de los resultados presentados es que hay diversas combinaciones de factores desfavorables que pueden conllevar en la realidad a situaciones, en las cuales es imposible encontrar un método factible para localizar instalaciones ilegales. Para los casos de conexiones ilegales directas a la red o derivaciones de conexiones vecinales, ello es la excepción a la regla. Sin embargo, este problema puede presentarse con mayor frecuencia para aquellos bypasses ubicados cerca de la tubería legal y los cuales presentan obstáculos que inhiben la introducción de cámaras endoscópicas. Por ello, en ciertos casos si será necesario realizar excavaciones en la búsqueda de estos en un área limitada alrededor de la caja portamedidor, si previamente se han descartado las otras opciones de hurtos.
Sin embargo, el desarrollo tecnológico continuo mejorará paulatinamente los métodos presentados como ayudará también a la aparición de nuevos métodos. Por ello el presente estudio no pretende presentar conclusiones definitivas sino presentar un avance y abrir de esta forma una discusión acerca de este tema de importancia en la gestión de las entidades prestadoras.

\section{Conclusiones}

Los cuatro métodos de localización de instalaciones ilegales analizadas son factibles dentro de los límites indicados.

El uso efectivo de equipos de localización de instalaciones ilegales depende de una serie de factores locales $y$ circunstanciales que deberían ser evaluados antes de adquirir y emplear los equipos. Independientemente de las circunstancias hay métodos que deberían ser descartados por ser de muy limitada aplicabilidad, ineficientes o no aplicables. Especialmente para el georradar y en menor grado también para el método electroacústico, la experiencia de los operarios de los equipos son claves para su aplicación exitosa. Con la tecnología disponible aquí analizada, solo con cámaras endoscópicas para conexiones se puede encontrar aquellos bypasses ubicados cerca de la tubería legal. Como no siempre son aplicables, en casos de sospecha de hurtos importantes, si será necesario realizar excavaciones alrededor de la caja portamedidor.

\section{Recomendaciones}

Para las entidades prestadoras que requieren realizar la localización de instalaciones ilegales se recomienda realizar análisis previos de la factibilidad 
de usar uno o varios de los métodos. Para ello se recomienda tomar en cuenta los factores siguientes: características del subsuelo, características de las redes e instalaciones domiciliarias, recursos humanos y económicos disponibles. En caso de dudas, la realización de pruebas puntuales o proyectos pilotos puede dar respuestas definitivas. Para incrementar el costo-beneficio de la localización de instalaciones ilegales se sugiere enfocar los esfuerzos en aquellos predios donde existe una sospecha previa de hurto independientemente del método empleado.

En la mayoría de las circunstancias locales, es recomendable una aplicación combinada de varios métodos de localización de instalaciones ilegales. Debido al hecho de que cierto tipo de instalaciones ilegales, bypasses cercanos a la tubería legal, son en muchas circunstancias imposibles de detectar, se hace recomendable la identificación de nuevas tecnologías para ello.

\section{Ageadecimientos}

Queremos agradecer a Ing. Lisbeth Quispe (SEDACUSCO S.A., Perú), Gregor Vitt (Hermann Sewerin GmbH, Alemania), Javier Rey (Sewerin Iberia, España), Henry Mallqui (H2O Instrument, Perú), Alberto Jiménez (Aqualogy/Suez, India), Mauricio Jiménez (GITEC Consult GmbH, Alemania), Henry Acevedo (Fluidis Servicios Asociados S.A.S., Colombia), Eloy Alarcón (AKUT PARTNER, Perú), Javier Carillo y Juan Carlos Mamani (EPS TACNA S.A., Perú) por sus importantes aportes. Finalmente queremos agradecer a la Cooperación Suiza SECO y la Cooperación Alemana al Desarrollo, implementada por la Deutsche Gesellschaft für Internationale Zusammenarbeit (GIZ) $\mathrm{GmbH}$, a través de PROAGUA II por haber hecho posible el presente estudio.

\section{Referencias}

Alva, T. y Ziemendorff S. Prevención $y$ detección de hurtos. Manual 4 de la serie "Manuales de Gestión Comercial". Lima: OTASS, 2019.

AQUALOGY Idroloc: Caracteristicas $y$ funcionalidad. Recuperado de: www.enerlogy.aqualogy.net/ $\mathrm{cl} /$ es/tecnologias-destacadas/ idroloc\#caracteristicas, 2014.

Doolittle, J.A.; Minzenmayer, F.E.; Waltman, S.W.; Benham, E.C.; Tuttle, J.W. y Peaslee, S.D. "Ground-penetrating radar soil suitability map of the conterminous
United States". Geoderma, Vol. 141, $\quad \mathrm{N}^{\circ}$ 3-4, (2007): 416421. DOI: doi.org/10.1016/j. geoderma.2007.05.015

Enright, James T. "Water Dowsing: The 'Scheunen' Experiments". Naturwissenschaften, Vol. 82, $\mathrm{N}^{\circ} 8$, (1995): 360-369.

Ziemendorff, S. "Detección de conexiones clandestinas de agua potable con métodos acústicos un nuevo método y su aplicación en campo". Revista INDES, Vol. 3, $\mathrm{N}^{\circ} 1$ (2015): 5-17 
Ziemendorff, S. y Perfecto O. "Comparación de técnicas de corte simple del servicio de agua potable". CAMPUS, Vol. 22, N 24 (2017): 229-250. DOI: doi.org/10.24265/ campus.2017.v22n24.08

Ziemendorff, S.; Perfecto O.; Mestanza, S. y Chuquizuta, C. "Comparación de técnicas de corte drástico del servicio de agua potable". CAMPUS, Vol. 23, № 25, (2018): 67-86. DOI: doi.org/10.24265/ campus.2018.v23n 25.06

Ziemendorff, S. "Medidas indicativas y preventivas contra la manipulación fraudulenta de medidores de agua". Tecnologia y Ciencias del Agua, Vol. $11, \mathrm{~N}^{\circ} 4(2020)$. 